\title{
A new insight into the cellular regulation of aqueous oufflow: how trabecular meshwork endothelial cells drive a mechanism that regulates the permeability of Schlemm's canal endothelial cells
}

\author{
J A Alvarado, R G Alvarado, R F Yeh, L Franse-Carman, G R Marcellino, M J Brownstein
}

Br J Ophthalmol 2005;89:1500-1505. doi: 10.1136/bjo.2005.081307

\begin{abstract}
Aim: To test the hypothesis that trabecular meshwork endothelial cells (TMEs) increase the permeability of Schlemm's canal endothelial cells (SCEs) by actively releasing ligands that modulate the barrier properties of SCEs.

Methods: The TMEs were first irradiated with a laser light and allowed to condition the medium, which is then added to SCEs. The treatment response is determined by both measuring SCE permeability (flow meters) and the differential expression of genes (Affymetrix chips and quantitative polymerase chain reaction (PCR)). The cytokines secreted by the treated cells were identified using ELISA and the ability of these cytokines to increase permeability is tested directly after their addition to SCEs in perfusion experiments.

Results: SCEs exposed to medium conditioned by the light activated TMEs (TME- $\mathrm{cm}$ ) respond by undergoing a differential expression (DE) of 1120 genes relative to controls. This response is intense relative to a DE of only 12 genes in lasered SCEs. The TME-cm treatment of SCEs increased the SCE permeability fourfold. The role of cytokines in these responses is supported by two findings: adding specific cytokines established to be secreted by lasered TMEs to SCEs increases permeability; and inactivating the TME-cm by boiling or diluting, abrogates these conditioned media permeability effects.

Conclusion: These experiments show that TMEs can regulate SCE permeability and that it is likely that TMEs have a major role in the regulation of aqueous outflow. This novel TME driven cellular mechanism has important implications for the pathogenesis of glaucoma and the mechanism of action of laser trabeculoplasty. Ligands identified as regulating SCE permeability have potential use for glaucoma therapy.
\end{abstract}

$\mathrm{T}$ he conventional aqueous outflow pathway (CAOP) performs the dual functions of facilitating the egress of aqueous from the anterior chamber of the eye into the lumen of Schlemm's canal and of preventing the reflux of blood from the venous circulation into the anterior chamber.' In carrying out these two tasks the CAOP is endowed with two endothelial cell barriers, which are specialised and positioned in series. As aqueous exits from the eye it first encounters the trabecular meshwork endothelial cells (TMEs) that line aqueous channels, and then subsequently encounters the endothelial cells that line the lumen of Schlemm's canal (SCEs). SCEs facilitate aqueous outflow by forming "giant vacuoles" only when the intraocular pressure (IOP) exceeds the pressure in the episcleral venous plexus. ${ }^{2}$ Similarly, SCEs discourage the reflux of blood by preventing the formation of giant vacuoles when the episcleral venous pressure exceeds the IOP. We surmise that TMEs have similar mechanisms but these are subtle and involve interactions with SCEs via the release of molecular factors and activities that have yet to be identified.

\section{METHODS}

\section{Cell culture, laser light treatment, and conductivity} measurements

The human cultured TMEs and SCEs used for our experiments are grown and maintained in cell culture conditions using previously described methods..$^{2-9}$ The principal treatments involve irradiating the cells with visible light ("lasered"), and adding media conditioned by the irradiated cells to untreated cells. Each experiment includes eight conditions in quadruplicate samples (or 32 preparations/ experiment), with the experiment lasting for 48 hours. Four of the eight conditions involve TMEs: control TMEs, lasered TMEs, TMEs treated by exposure to medium conditioned by lasered TMEs (TME-cm), and SCEs exposed to the TME-cm. The other four involve SCEs: control SCEs, lasered SCEs, SCEs treated by exposure to medium conditioned by lasered SCEs (SCE-cm), and TMEs exposed to the SCE-cm. The preparations are lasered using a frequency doubled, Q switched Nd:YAG (F-D N:Y) instrument set to deliver a standard number of low fluence pulses $(0.8 \mathrm{~mJ} /$ pulse or $600 \mathrm{~mJ} / \mathrm{cm}^{2}$ ). The laser beam measures $400 \mu \mathrm{m}$ in diameter with a wavelength of $532 \mathrm{~nm}$ and a pulse duration of $\sim 3$ ns. ${ }^{10-13}$ The 32 preparations in each experiment have the conductivity measured in $\mu \mathrm{l} / \mathrm{min} / \mathrm{mm} \mathrm{Hg} / \mathrm{cm}^{2},{ }^{2}{ }^{14}$ and other assays performed as described below.

\section{Gene chip assays, quantitative PCR, and ELISA}

We assess the responses to the laser and media exposure treatments by determining the gene expression profiles (Affymetrix gene chips), and verifying these results using quantitative PCR. The synthesis of proteins corresponding to detected differential expressed genes is determined using ELISA. Total RNA is extracted with Trizol (Invitrogen, Carlsbad, CA, USA), and cDNA samples are prepared using the NuGEN Technologies Ovation RNA amplification and Biotin Labeling system (Version 1.0) according to the

Abbreviations: $C A O P$, conventional aqueous outflow pathway; $D E$, differential expression; DEGs, differentially expressed genes; ELISA, enzyme linked immunosorbent assay; F-D N:Y, frequency doubled, $Q$ switched Nd:YAG; IL, interleukin; IOP, intraocular pressure; PCR, polymerase chain reaction; POAG, primary open angle glaucoma; RMA, robust multiarray average; SCEs, Schlemm's canal endothelial cells; TMEs, trabecular meshwork endothelial cells; TNF, tumour necrosis factor 


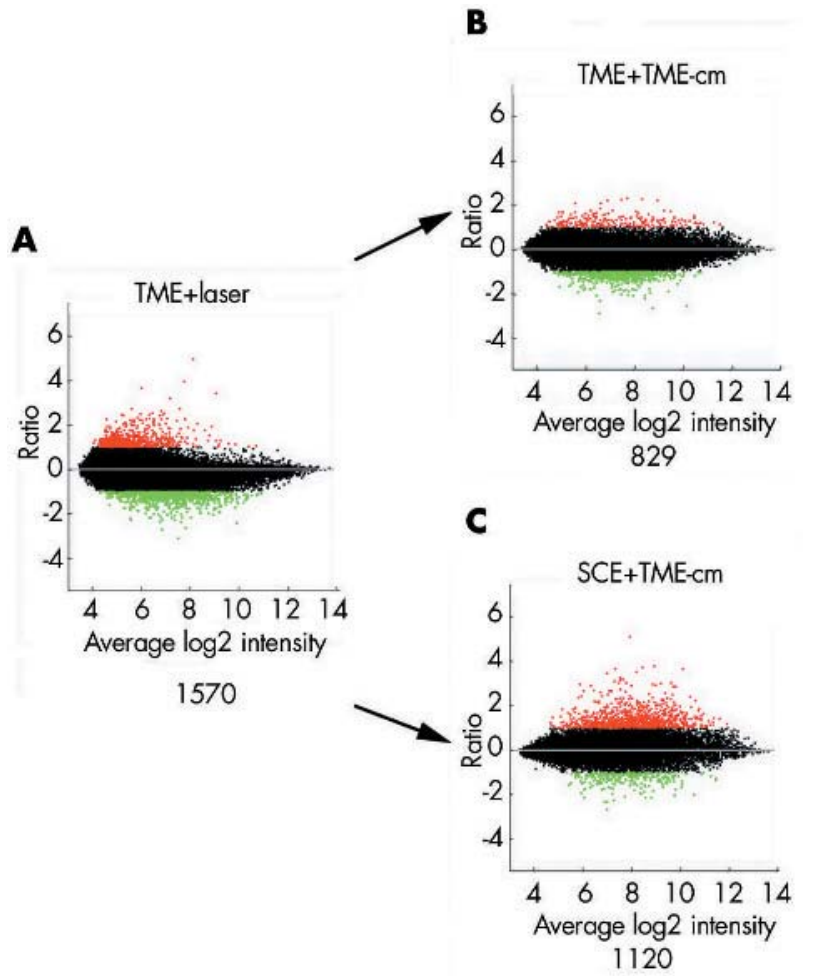

Figure 1 Ratio intensity scatter plot comparing the profile of genes expressed by TMEs treated by the application of short pulse/green light delivered by a frequency doubled Nd:YAG laser instrument $(\mathrm{A})$; by the addition of media conditioned by the irradiated TMEs (TME-cm) when added to naive TMEs (B); or by adding TME-cm to naive SCEs (C). Each dot represents the mean intensity log ratio (base 2) versus the average log intensity of a single gene based on four replicas (that is, one chip for each of four samples), with each replicate containing 11 probes/gene, using approximately 47000 transcripts from 38500 well characterised genes. Red and green dots indicate upregulated and downregulated genes, respectively, demonstrating a twofold or greater differential expression (DE) ratio with the total number of differentially expressed genes (DEGs) for each category indicated below each graph.

manufacture's instructions for the indicated amount of starting RNA (5-100 ng) as previously reported. ${ }^{15}$ For sscDNA microarrays, all samples were placed in standard Affymetrix hybridisation buffer and the arrays were stained with phycoerythrin-streptavidin according to manufacture's recommendations. ${ }^{16}{ }^{17}$ Real time quantitative PCR was used to measure the expression of selected mRNAs using a 5'fluorogenic nuclease assay on the ABI Prism 7900 following manufacturer's instructions (Applied Biosystems, Foster City, CA, USA). Enzyme linked immunosorbent assay (ELISA) is used to identify secreted cytokines by disrupting the cells using a lyses buffer containing 1.0\% NP-40, $150 \mathrm{mM} \mathrm{NaCl}$, and $50 \mathrm{mM}$ of TRIS at $\mathrm{pH} 8.0$ following instructions from the ELISA kit manufacturer.

\section{Statistical analysis for array data}

The raw image data are analysed using GeneChip Expression Analysis Software (Affymetrix or affy) to extract perfect match and mismatch values, to which we apply the robust multiarray average (RMA) algorithm ${ }^{18}{ }^{19}$ implemented in the Bioconductor/affy package ${ }^{20}$ under the free statistical computing environment $\mathrm{R}$ (www.r-project.org) to background correct, quantile normalise, and summarise values of 11 probe pairs for each gene (probe set) on each chip. This results in a $54675 \times 32$ matrix of logarithm based 2 of gene expression measures, where columns correspond to different gene chips and rows correspond to the different genes (probe

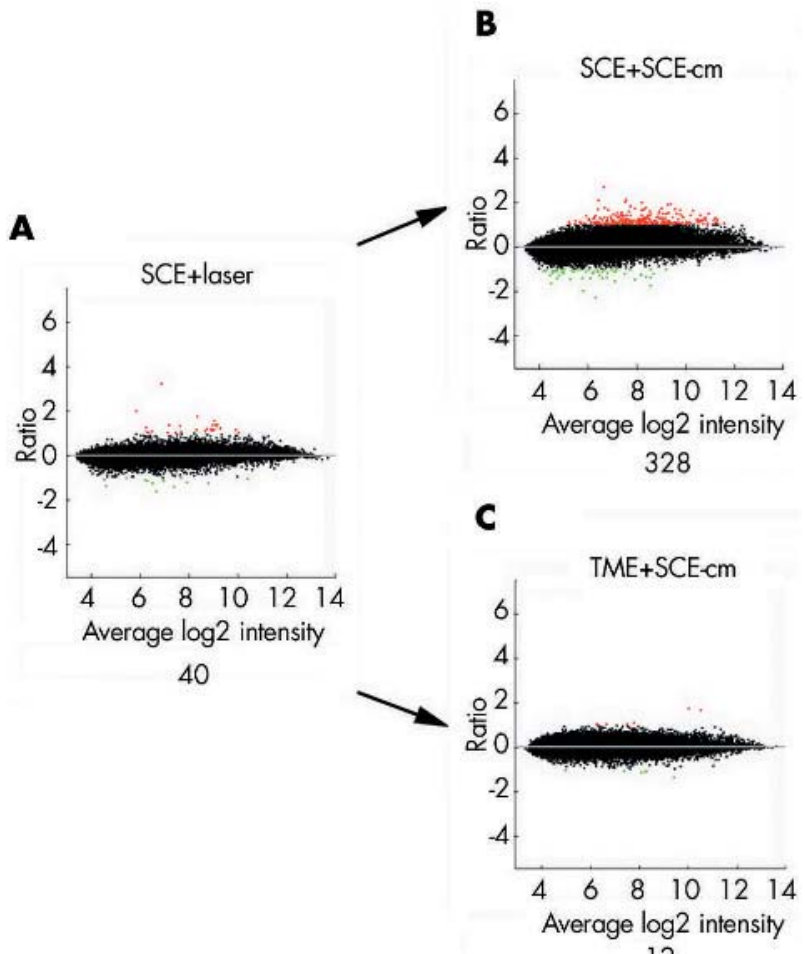

12

Figure 2 Ratio intensity scatter plot as in figure 1 showing profiles DEGs in SCEs after the lasering procedure (A); or after adding SCE-cm to naive SCEs (B); or after adding SCE-cm to naive TMEs (C).

sets). For a typical gene (probe set), we have four replicate expression measures (from four chips) for each of the eight conditions as described above.

\section{RESULTS \\ Differentially expressed genes (DEGs) in treated/ untreated preparations}

Genes with a twofold or greater expression relative to controls are considered differentially expressed (DEGs). DEGs are shown in figures 1 and 2, which are MA ratio intensity scatter plots where " $\mathrm{A}$ " represents the average of log intensities across the 32 chips in each experiment, and " $\mathrm{M}$ " represents the ranking of genes according to the strength of evidence of differential expression. In both figures upregulated DEGs are shown as red dots and downregulated genes as green dots. Figure 1 shows the elicited response relative to the respective controls for irradiated TMEs (fig 1A), for medium prepared by irradiated TMEs when added to other TMEs (fig 1B), or when added to SCEs (fig 1C). Figure 2 shows the response elicited for irradiated SCEs (fig 2A), for medium prepared by irradiated SCEs when added to other SCEs (fig $2 \mathrm{~B}$ ), or when added to TMEs (2C).

Comparing both figures shows that the red and green dots are far more numerous in figure 1 than in figure 2 . The actual counts of the number of DEGs for the six functional categories in both figures, shown below each panel in these figures, support this impression. In figure 1 the DEG counts are approximately three orders of magnitude for all three conditions, whereas in figure 2 the counts are one or two orders of magnitude for the same three conditions. Thus, across all functional categories, we find the number of DEGs is much greater for TMEs than for SCEs. Not only are TMEs more responsive to the laser treatment than are SCEs, but also media conditioned by TMEs are more effective for 
A

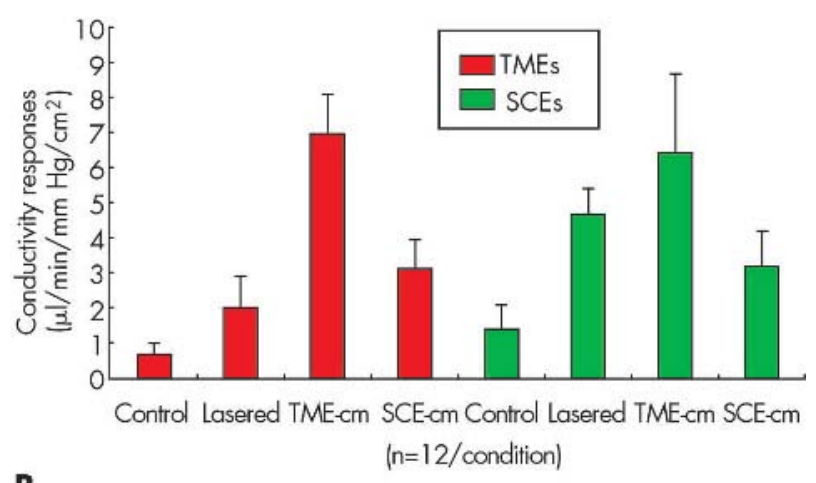

B

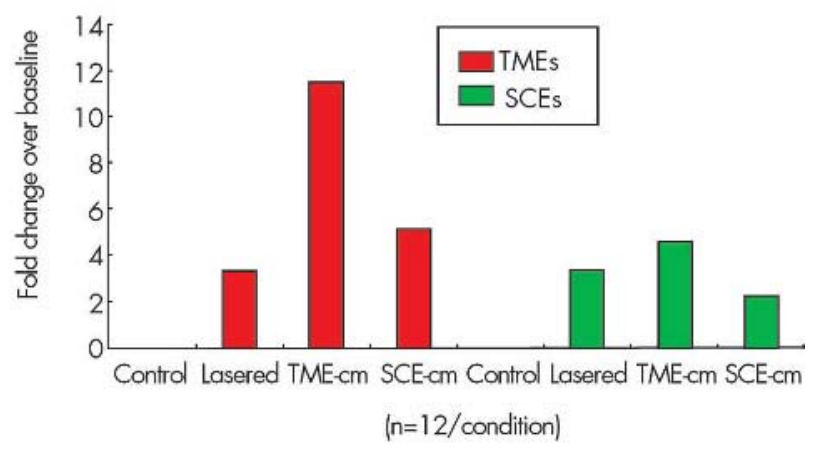

Figure 3 (A) The conductivity responses in $\mu \mathrm{l} / \mathrm{min} / \mathrm{mm} \mathrm{Hg} / \mathrm{cm}^{2}$, with the mean (SD), measured in monolayers of both TMEs and SCEs in controls and in treated preparations as indicated. In (B) the treatment responses are depicted adjusting for differences in baseline as a fold change. Note that in all cases, the responses are robust, amounting to increases of over 11-fold in the case of the TME-cm, the highest response, and $200 \%$ in the case of naive SCEs treated with SCE-cm, the smallest response.

stimulating both SCEs and TMEs than are media conditioned by SCEs.

\section{Conductivity in treated/untreated preparations}

Figure 3A reports the means (SD) of the conductivity (C) responses measured for the eight experimental conditions tested for both TMEs and SCEs. The means for the six treatment conditions differ significantly from the control means $(p \leqslant 0.001)$. Figure 3B depicts the fold changes in these same C responses after correcting for baseline differences. It can be seen that for both TMEs and SCEs, the largest increases in C occur when the cells are treated by exposure to medium conditioned by lasered TMEs (TME$\mathrm{cm})$. These $\mathrm{C}$ responses are congruous, condition by condition, with the results of the gene expression experiments showing the TME- $\mathrm{cm}$ to be a potent inducer of DEGs. These results are consistent with our original hypothesis that medium conditioned by TMEs increases SCE permeability. In fact, the permeability increases fourfold in SCEs exposed to the TME-cm.

\section{Controls}

The media conditioned by laser activated TMEs (TME-cm) was boiled for 5 minutes or diluted five times by the addition of fresh cell culture media to inactivate any factors released into the extracellular environment. When these "control" media, or medium prepared by TMEs without previous lasering, were added to TMEs, C remains at baseline level, while that from the laser activated TMEs produces the expected increase in $\mathrm{C}$, as shown in figure 4 .

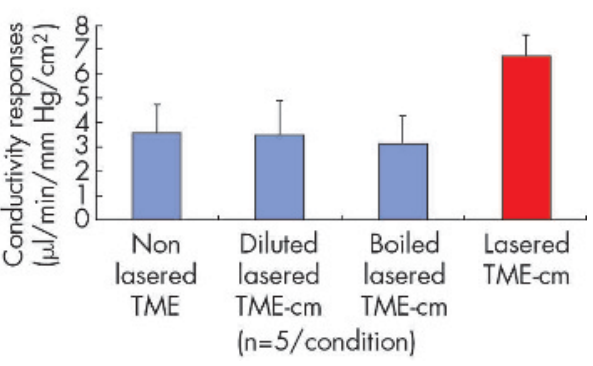

Figure 4 Conductivity responses measured in $\mu \mathrm{l} / \mathrm{min} / \mathrm{mm} \mathrm{Hg} / \mathrm{cm}^{2}$ showing the mean and SD in controls (blue) and in TMEs treated by the addition of media conditioned by light irradiated TMEs (red). Adding media from naive TMEs, or from laser activated TMEs that had been inactivated by dilution or boiling effectively abrogated the increase in conductivity (blue), relative to the situation when media conditioned by light irradiated TMEs was added (red).

\section{Identification of cytokines secreted by TMEs}

The gene for the chemokine interleukin 8 (IL-8) is DE relative to controls in lasered TMEs and SCEs $(\mathrm{p}<0.01)$ and in SCEs treated with TME-cm $(\mathrm{p}<0.001)$. The DE of IL- 8 was verified using Q-PCR assays (fig 5A) which shows that the IL-8 gene is DE in lasered TMEs $(p<0.05)$, TMEs exposed to media conditioned by SCEs $(\mathrm{p}<0.01)$, SCEs exposed to the TME-cm $(p<0.05)$, and SCEs exposed to the SCE-cm $(p<0.01)$. Figure 5B shows that the protein coded by the IL- 8 gene is clearly secreted at higher levels relative to controls in five out of the six conditions tested $(\mathrm{p}<0.01)$. Figure $5 \mathrm{C}$ presents the same data as in figure $3 \mathrm{~A}$ in order to compare the conductivity data with those for the IL-8 protein synthesis in figure 5B. Similar responses are apparent for five of the six conditions tested in the conductivity and protein synthesis experiments. Lasered TMEs are activated at the gene level to express the appropriate transcripts for a given cytokine, signals that lead to the synthesis of the corresponding mRNAs and, ultimately, to the synthesis and release of the chemokine IL-8. Importantly, these responses occur in a manner congruous with the conductivity increases observed in the various experimental conditions. By noting DEGs among the 298 representing all known cytokines and chemokines, we should be able to identify those cytokines that are potentially synthesised by irradiated TMEs.

Other ELISA experiments (data not shown) indicate that lasered TMEs release interleukin $1 \alpha$ (IL-l $1 \alpha)$, interleukin $1 \beta$ (IL-1 $\beta)$, and tumour necrosis factor alpha (TNF- $\alpha$ ) into the medium. The cytokine release process occurs in a doseresponse relation: increasing the power from $0.1 \mathrm{~mJ}$ to $1.0 \mathrm{~mJ}$, while holding the number of laser shots constant, induces lasered TMEs to respond by releasing increasing quantities of TNF- $\alpha$.

\section{Conductivity response of SCEs by adding four cytokines}

Adding IL- $1 \alpha$, IL- $1 \beta$, TNF- $\alpha$, and IL- 8 directly to monolayers of SCEs, we examined the ability of these cytokines to increase SCE permeability. Figure 6 shows that an increase in conductivity relative to controls was measured in all of the four factors tested added at a given concentration. Dose/ response curves will be obtained in future studies to compare the potency of each agent. However, inspection of this graph shows that the four factors induce substantial increases in the SCE conductivity. These data support the concept that the conductivity effects induced by the TME-cm when added to SCEs are likely mediated by media borne factors as postulated in our hypothesis. 
A

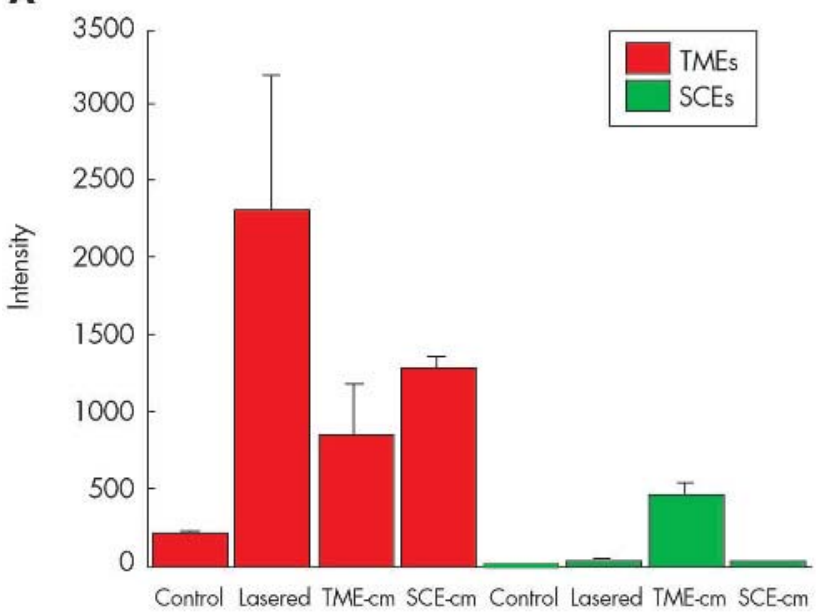

B

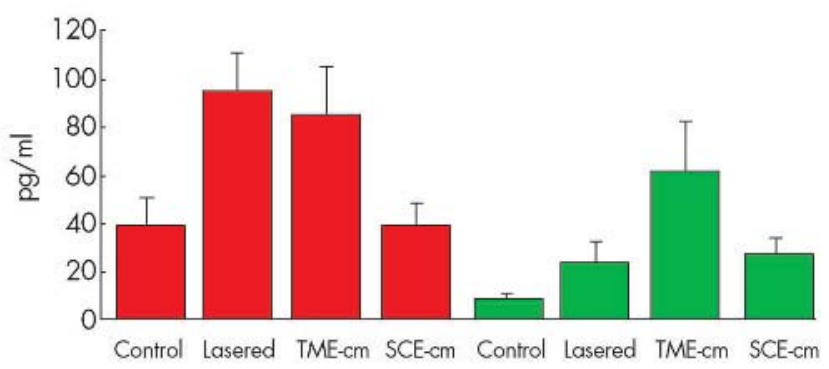

C

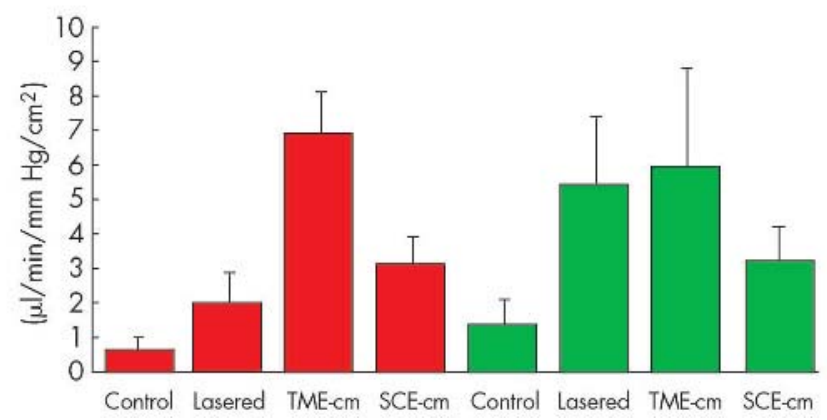

Figure 5 Intensity plot depicting the mRNA responses (means (SD)) measured using quantitative PCR (Q-PCR) in the eight experimental and control preparations (A), and at the protein level measured using ELISA (B). These responses are correlated with those measured in conductivity assays (C). The mRNA responses are measured as the mean intensity log ratio (base 2). The ELISA measurements are expressed in pg/ml, and the conductivity studies units in $\mu \mathrm{l} / \mathrm{min} / \mathrm{mm} \mathrm{Hg} / \mathrm{cm}^{2}$.

\section{DISCUSSION}

Our experiments have provided substantial support for the hypothesis that TMEs release factors into the media, and that these factors upon binding to SCEs increase the permeability of the SCE barrier. SCEs form the last cellular barrier

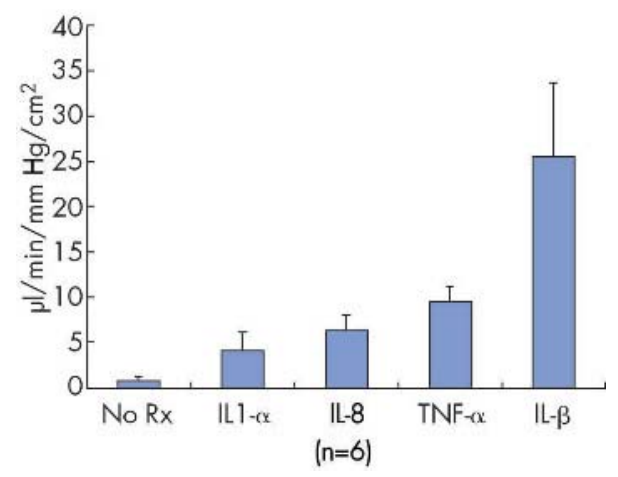

Figure 6 Plot showing changes in conductivity induced by the direct addition to SCE monolayers of $10 \mathrm{ng}$ of ILI- $\alpha, 5 \mathrm{ng}$ of IL-8, $15 \mathrm{ng}$ of TNF- $\alpha$, and $10 \mathrm{ng}$ of IL- $\beta$ ? . Mean (SD) are shown. traversed by aqueous as it exits from the eye and enters into the venous circulation. As such the SCE barrier is strategically located to act as a "control" site, so that increasing the permeability of this barrier augments the egress of aqueous from the eye. TMEs, according to our findings, drive a mechanism controlling the SCE permeability by releasing vasoactive cytokines and other factors that have the capacity to increase the permeability of the SCE barrier. In fact, the addition of media conditioned by the irradiated TMEs to monolayers of untreated SCEs results in a $400 \%$ increase in SCE conductivity. The involvement of media borne factors secreted by TMEs has been addressed directly in several ways. In one set of experiments we demonstrated that in irradiated TMEs the gene for IL- 8 is upregulated, and that the corresponding mRNAs undergo a congruous induction, resulting in the synthesis of the IL-8 protein. In another set of experiments, we demonstrated that three other cytokines are released into the media by TMEs as a function of the number of laser pulses applied and the power used. When each of these four cytokines is added individually to SCEs, the conductivity increased as we have postulated. The role of media factors is also supported by control studies showing that boiling, diluting, or using medium from untreated TMEs, abrogates the TME medium effects on SCE permeability. 
Our findings highlight the importance of the well differentiated endothelial cells of the trabecular meshwork and Schlemm's canal as a system in which to study cell-cell interactions. These interactions are complex and proceed in both directions, involving TME-SCE and SCE-TME relations, as well as mutual exchanges concerned with TME-TME and SCE-SCE associations. For example, we have learned that the responses to light irradiation treatment are specific for each cell type, with irradiated TMEs undergoing a DE of 1570 genes compared to irradiated SCEs, which undergo a DE of only 40 genes. Similarly, the responses to the addition of media conditioned by irradiated cells are specific to each cell type. Treatment by the addition of media conditioned by irradiated TMEs to either untreated TMEs or SCEs induces the DE of 829 and 1120 genes respectively (total of 1949). In comparison, medium conditioned by laser activated SCEs when added to either SCEs or TMEs induces only 328 and 12 DE genes (total of 340). Thus, there is at least a 500\% greater induction of DE genes by TME conditioned medium compared to SCE conditioned medium. These differences are reflected as well by the conductivity increases induced by each cell type: medium conditioned by the lasered TMEs induces a $200 \%$ greater increase in conductivity compared to medium conditioned by the lasered SCEs when added to TMEs and SCEs.

The finding that cytokines released by TMEs regulate the permeability of the SCE barrier is novel. It is not readily apparent how this TME driven mechanism contributes to the two functions of the conventional aqueous outflow pathway facilitating aqueous outflow and preventing the reflux of blood. We propose that a mechanism exists for TMEs, which is essentially similar to that described in the introduction driving the formation of giant vacuoles by SCEs. The trabecular meshwork and the lining TMEs, as well as Schlemm's canal and the lining SCEs, undergo deformation and stretching with changes in intraocular pressure. ${ }^{21}$ Stretching TMEs by mechanical means or by increasing the IOP elicits a wide variety of important biochemical responses. ${ }^{22-25}$ Assuming that TMEs have stretch receptors, the increased tension makes the trabecular beams and cords taut, thus triggering the stretch receptors to activate TMEs to release vasoactive factors that will increase flow across SCEs. When the IOP is less than the venous pressure, the beams and cords become flaccid, thus increasing the resistance presented by SCEs.

If such a tension sensitive mechanism does in fact exist, it could provide a biological basis for the action of miotics during glaucoma therapy. For example, miotics like pilocarpine, upon inducing contraction of the ciliary muscle, also increase tension along the trabecular meshwork beams and cords, which in turn activates the stretch receptor to turn on the TMEs. TMEs, under the influence of the miotic mediated increase in tension, would then release the factors required to increase SCE conductivity, and thus the egress of aqueous. We propose that the interaction of mechanical effects generating tension and biological mechanisms releasing vasoactive factors work together during the miotic mediated increase in aqueous outflow, and perhaps even during accommodation.

In primary open angle glaucoma (POAG), trabecular meshwork endothelial cells are markedly decreased relative to those of age matched normals. ${ }^{26-30}$ This progressive decline in cell density results in a loss of $\sim 0.58 \%$ cells/year, which is most pronounced in the inner layers of the trabecular meshwork's filtration zone. The inner trabecular cells may be prone to injury by free oxygen radicals carried in the aqueous outflow. ${ }^{29}$ When we first noticed the loss of TMEs in POAG, it was difficult to comprehend how this cell loss could have a negative impact on the facility of aqueous outflow and the pathogenesis of glaucoma, ${ }^{26}$ particularly in view of the generally held concept that the greatest resistance to aqueous outflow is presented by the SCEs. ${ }^{31}$ Recent evidence demonstrates that there is extensive oxidative DNA damage involving the trabecular cells of patients with POAG, affecting the filtration zone and inner trabecular meshwork layers. ${ }^{32}$ Other studies report that incorporating a specific type of myocilin mutant known to be present in vivo in certain types of open angle glaucoma into TMEs in vitro (that is, Pro370Leu) results in "killing" of cultured human TMEs. ${ }^{33}$ The demise of TMEs is the result of misfolding, aggregation, and build up of this protein in the endoplasmic reticulum of trabecular cells. ${ }^{33}$ Whether caused by oxidative DNA damage or abnormal processing of protein folding by TMEs in POAG, there is significant evidence that the normal population of trabecular meshwork cells is affected by dysfunction and death. In view of our findings, it is clear how such a loss of trabecular meshwork cells, by reducing the quantity of cytokines released by a diminished population of TMEs, could have a negative impact on the homeostasis of aqueous outflow. The reduced load of cytokines and other factors may not maintain the porosity of SCEs necessary to facilitate aqueous outflow and the IOP may rise to the abnormal levels characteristic of many patients with glaucoma.

Our results provide a new understanding of the mechanism of action of glaucoma laser therapy using the F-D N:Y laser, based on the activities of TMEs and SCEs. ${ }^{34}{ }^{35}$ In view of the fact that lasering effects appear to be cell specific, we suggest a more prominent role for TMEs, which are most intensely activated by F-D N:Y laser treatment. The laser treatment is proposed to function by intensely activating the TMEs, which release cytokines that flow downstream and bind to SCEs. The SCEs, by undergoing a decrease in resistance, allow the more rapid egress of aqueous from the eye. It is important to recall that TMEs release matrix metalloproteinases, which by promoting fluid flow across the extracellular matrix, also participate in facilitating the overall rate of aqueous outflow. ${ }^{36}$ The interactions between TMEs and SCEs proceed in both directions, and involve relations within cells of a given type. Interactions between TMEs allow for these cells to release factors that would affect the TMEs lining the outermost aqueous channels, which must be crossed before aqueous can pass into the juxtacanalicular tissues. The paracellular route of the outer TMEs is more porous than that of TMEs in other regions. Perhaps this widening of the paracellular route of TMEs lining the outermost trabecular meshwork is related to the cumulative effects of cytokines released by the entire population of TMEs becoming most concentrated, and having the greatest effect in TMEs near the juxtacanalicular tissues. Although SCEs are less activated, factors released by these cells are particularly potent in promoting transendothelial flow across SCEs, as demonstrated by our experiments. We conclude that the use of the F-D Nd:Y laser, and the in vitro methods described, has allowed us to identify four cytokines released by lasered TMEs. Completion of this survey of the known 298 cytokines/ chemokines is a realistic goal and such knowledge may enhance our future ability to manipulate aqueous outflow during glaucoma therapy.

\section{ACKNOWLEDGEMENTS}

This research is supported by a major grant from the Thomas $\mathrm{J}$ Long Foundation, The Peninsula Community Foundation, That Man May See, Inc, the Department of Ophthalmology at UCSF, Research to Prevent Blindness, Coherent Inc, Lumenis Inc, the Joan Leidy Foundation, and contributions from many generous patients of JA Alvarado. The editorial assistance of Bryan Winn, MD, Richard P Juster, PhD, David Hollander, MD, and JAA's devoted and capable administrative assistant, Monica Lee, is gratefully acknowledged. 


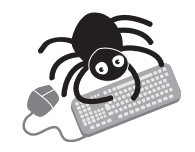

See the BJO website (www.bjophthalmol.com/supplemental) for the unabridged version of this paper.

\section{Authors' affiliations}

J A Alvarado, R G Alvarado, L Franse-Carman, Department of Ophthalmology, University of California San Francisco, CA 94143, USA R-F Yeh, Department of Epidemiology and Biostatistics, University of California San Francisco, CA 94143, USA

G R Marcellino, OptiMedica Corporation, Santa Clara, CA 95050, USA M J Brownstein, The J Craig Venter Institute, Rockville, MD, USA

This paper was presented at the Annual Meeting of the American Ophthalmological Society (AOS) in Sea Island, Ga, USA on 24 May 2005 and will be published digitally in the Proceedings of the AOS in December 2005. For the unabridged presentation of this paper see the BJO Online website. All work was performed at the University of California San Francisco, CA, USA.

Correspondence to: Professor Jorge A Alvarado, University of California San Francisco; Department of Ophthalmology; 10 Koret Way; San Francisco, CA 94143-0730, USA; jalva@itsa.ucsf.edu

Accepted for publication 19 August 2005

\section{REFERENCES}

1 Hogan M, Alvarado J, Weddell J. Histology of the human eye: an atlas and textbook. Philadelphia: WB Saunders, 1971:1-687.

2 Alvarado J, Betanzos A, Franse-Carman L, et al. Endothelia of schlemm's canal and trabecular meshwork: distinct molecular, functional, and anatomic features. Am J Physiol Cell Physiol 2004:286:C621-34.

3 Alvarado J, Franse-Carman L, McHolm G, et al. Epinephrine effects on major cell types of the aqueous outflow pathway: in vitro studies/clinical implications. Trans Am Ophthalmol Soc 1990;88:267-88.

4 Alvarado J, Franse-Carman L, McHolm G, et al. The response of the meshwork cells to adrenergic agents and their antagonists. In: Krieglstein, ed. Glaucoma update IV. Heidelberg: Springer Verlag, 1991:9-19.

5 Alvarado JA, Murphy CM, Franse-Carman L, et al. Effect of beta-adrenergics on paracellular width and 'fluid flow across outflow pathway cells. Invest Ophthalmol Vis Sci 1998;39:1813-22.

6 Alvarado JA, Wood I, Polansky JR. Human trabecular cells. II. Growth pattern and ultrastructural characteristics. Invest Ophthalmol Vis Sci 1982;23:464-78.

7 Underwood JL, Murphy GM, Chen J, et al. Glucocorticoids regulate transendothelial fluid flow resistance and formation of intercellular junctions. Am J Physiol 1999:277:C330-42.

8 Yun AJ, Murphy CG, Polansky JR, et al. Proteins secreted by human trabecular cells. Glucocorticoid effects. Invest Ophthalmol Vis Sci 1989;30:2012-22.

9 Polansky JR, Weinreb RN, Baxter JD, et al. Human trabecular cells: I. Establishment in tissue culture and growth characteristics. Invest Ophthalmol Vis Sci 1979:18:1043-9.

10 Latina $M$, Park $C$. Selective targeting of trabecular meshwork cells: in vitro studies of pulsed and cw laser interactions. Exp Eye Res 1995;60:359-72.

11 Latina M, Flotte T, Crean E, et al. Immunohistochemical staining of the human anterior segment. Arch Ophthalmol 1988;106:95-9.
12 Damji K, Shah K, Rock W, et al. Selective laser trabeculoplasty versus argon laser trabeculoplasty: a prospective randomized clinical trial. $\mathrm{Br} J \mathrm{Ophthalmol}$ 1999;83:718-22

13 Anderson R, Parrish J. Selective photothermolysis: precise microsurgery by selective absorption of pulsed radiation. Science, New Series 1983;220:524-7.

14 Perkins TW, Alvarado JA, Polansky JR, et al. Trabecular meshwork cells grown on filters. Conductivity and cytochalasin effects. Invest Ophthalmol Vis Sci 1988;29:1836-46.

15 Barczak A, Rodrigues M, Hanspers K, et al. Spotted long oligonucleotide arrays for human gene expression analysis. Genome Res 2003;13:1775-85.

16 Ginzinger $D$. Gene quantification using real-time quantitative PCR: an emerging technology hits the mainstream. Exp Hematol 2002;30:502-12.

17 Livak K, Schmittgen T. Analysis of relative gene expression data using realtime quantitative pcr and the 2(-delta delta $\mathrm{c}(\mathrm{t}))$ method. Methods Mol Biol 2001;25:402-8.

18 Bolstad B, Irizarry R, Astrand M, et al. A comparison of normalization methods for high density oligonucleotide array data based on variance and bias. Bioinformatics 2003:19:185-93

19 Irizarry R, Bolstad B, Collin F, et al. Summaries of affymetrix genechip probe level data. Nucleic Acids Res 2003;31:e15.

20 Gentleman R, Carey V, Bates D, et al. Bioconductor: open soffware development for computational biology and bioinformatics. Genome Biol 2004;5:R80.

21 Johnstone M, Grant WM. Pressure-dependent changes in structures of the aqueous outflow system of human and monkey eyes. Am J Ophthalmol 1973;75:365.

22 Mitton K, Tumminia S, Arora J, et al. Transient loss of $\alpha$ B-crystallin: an early cellular response to mechanical stretch. Biochem Biophys Res Commun 1997;235:69-73.

23 Tumminia S, Mitton K, Arora J, et al. Mechanical stretch alters the actin cyłoskeletal network and signal transduction in human trabecular meshwork cells. Invest Ophthalmol Vis Sci 1998:39:1361-71.

24 Gonzalez P, Epstein D, Borras T. Gene up-regulated in the human trabecular meshwork in response to elevated intraocular pressures. Invest Ophthalmol Vis Sci 2000;41:352-61.

25 Borras T, Rowlette L, Tamm E, et al. Effects of elevated intraocular pressure on oufflow facility and TIGR/MYOC expression in perfused human anterior segments. Invest Ophthalmol Vis Sci 2002;4:33-40.

26 Alvarado JA, Murphy CG. Oufflow obstruction in pigmentary and primary open angle glaucoma. Arch Ophthalmol 1992;110:1769-78.

27 Alvarado J, Murphy C, Polansky J, et al. Age-related changes in trabecular meshwork cellularity. Invest Ophthalmol Vis Sci 1981;21:714-27.

28 Alvarado J, Murphy C, Juster R, et al. Studies on the pathogenesis of primary open-angle glaucoma: Regional analyses of trabecular meshwork cellularity and dense collagen. In: David R, ed. Recent advances in glaucoma. Amsterdam: Excerpta Medica, 1984:3-8.

29 Alvarado J, Murphy C, Juster R. Trabecular meshwork cellularity in primary open-angle glaucoma and nonglaucomatous normals. Ophthalmology 1984;91:564-79.

30 Murphy CG, Johnson M, Alvarado JA. Juxtacanalicular tissue in pigmentary and primary open angle glaucoma. The hydrodynamic role of pigment and other constituents. Arch Ophthalmol 1992;110:1779-85.

31 Epstein DL, Rohen JW. Morphology of the trabecular meshwork and innerwall endothelium after cationized ferritin perfusion in the monkey eye. Invest Ophthalmol Vis Sci 1991;32:160-71.

32 Sacca SC, Pascotto A, Camicione $P$, et al. Oxidative DNA damage in the human trabecular meshwork. Arch Ophthalmol 2005;123:458-63.

33 Liu Y, Vollrath D. Reversal of mutant myocilin non-secretion and cell killing: implications for glaucoma. Human Mole Genetics 2004;13:1193-204.

34 Van Buskirk E, Pond V, Rosenquist R, et al. Argon laser trabeculoplasty. Studies of mechanism of action. Ophthalmology 1984;91:1005-10.

35 Van Buskirk E. Pathophysiology of laser trabeculoplasty. Surv Ophthalmol 1989;33:264-72

36 Acott T, Wirtz M. Biochemistry of aqueous oufflow. In: Ritch R, Shields M, Krupin T, eds. The glaucomas. 2nd ed. St Louis: Mosby, 1996:281-305. 